Agriculture et systèmes alimentaires face à la Covid-19/ Agriculture and Food Systems in the face of COVID-19 Disponible en ligne : Coordonnateurs : Patrick Dugué, Mohamed Taher Sraïri, Jean-Yves Jamin www.cahiersagricultures.fr

\title{
Crises environnementales et sanitaires: des maladies de l'anthropocène qui appellent à refonder notre système alimentaire
}

\author{
Michel Duru* et Claire Le Bras \\ INRAE, UMR 1248 AGIR, Université Toulouse, INPT, 31326 Castanet Tolosan, France
}

\begin{abstract}
Résumé - Depuis le milieu du $\mathrm{XX}^{\mathrm{e}}$ siècle, les impacts des activités humaines sur les écosystèmes sont croissants. À l'érosion de la biodiversité et au dérèglement climatique, ainsi qu'au développement de maladies chroniques que constituent l'obésité et le diabète, s'ajoute désormais la pandémie du coronavirus. Il s'agit d'un ensemble de crises environnementales ou sanitaires qui résultent pour partie de facteurs communs et dont les impacts peuvent se conjuguer et s'amplifier. Dans ce contexte inédit, nos modes de production, transformation, distribution et consommation des aliments sont particulièrement interrogés. Ils sont à l'origine d'une part importante des émissions de gaz à effet de serre, participent à la destruction de certains habitats naturels réservoirs d'agents pathogènes et contribuent à l'émergence de maladies chroniques chez l'homme. De ce fait, la nécessité d'une transition de notre système alimentaire est une idée qui fait consensus, même si le choix des changements à opérer concrètement pose de nombreuses questions. Â travers une approche systémique de «santé globale», rendant compte de l'interdépendance de l'état de santé de l'Homme, des animaux et des écosystèmes dans lesquels ils évoluent, nous montrons qu'il faut prioriser aussi bien les enjeux environnementaux que de santé pour mener à bien ces arbitrages. Nous montrons qu'il est possible de faire des choix doublement vertueux pour l'environnement et la santé en transformant les modes de production, de transformation, de distribution et de consommation des aliments : réorienter l'élevage, abaisser le degré de transformation des aliments, diversifier les modes de distribution et «végétaliser» notre assiette. Ces changements participent à la territorialisation du système alimentaire.
\end{abstract}

Mots clés : agroécologie / changement climatique / déforestation / élevage / une seule santé

\begin{abstract}
Environmental and health crises: Anthropocene diseases that call for redesigning our food system. Since the middle of the 20th century, the impacts of human activities on ecosystems have been increasing. The erosion of biodiversity and climate change, as well as the pandemics of obesity and diabetes, are now compounded by the coronavirus pandemic. This is a set of environmental or health crises resulting in part from common factors and whose impacts can be combined and amplified. In this new context, our methods of food production, processing, distribution and consumption are particularly questioned. They are the source of a significant share of greenhouse gas emissions, participate in the destruction of some natural habitats which are reservoirs of pathogens, and contribute to the emergence of human chronic diseases. As a result, the need for a transition in our food system is a shared idea, even if the choice of the changes to be made concretely raises many questions. Through a systemic approach to "global health", reflecting the interdependence of the state of health of humans, animals, and the ecosystems in which they evolve, we show that synergies must be found between practices allowing to face environmental and health challenges. We show that it is possible to make doubly virtuous choices for the environment and health by transforming the modes of production, processing, distribution and consumption of food: reorienting animal husbandry, lowering the degree of food processing, diversifying distribution methods and increasing plant food. These changes contribute to set up territorialized food systems.
\end{abstract}

Keywords: agroecology / climate change / deforestation / livestock / one health

\footnotetext{
*Auteur de correspondance : michel.duru@inrae.fr
} 


\section{Une approche de santé globale pour} mieux appréhender les liens entre système alimentaire et crises environnementales et sanitaires

En moins d'un siècle, nous sommes entrés dans l'ère de l'anthropocène: nos activités, dont celles liées à notre système alimentaire, sont devenues une cause majeure de l'évolution de notre environnement (Gordon et al., 2017). Globalement, l'augmentation de la production agricole a permis de fournir assez de calories pour nourrir le monde mais a aussi généré des impacts particulièrement néfastes à l'échelle locale : érosion de la biodiversité, dégradation de la qualité des sols et de l'eau (Ipes Food, 2019), mais aussi à l'échelle mondiale: épuisement des énergies fossiles et du phosphore et surtout dérèglement climatique. Dans le même temps, la mortalité liée aux infections n'a cessé de diminuer grâce aux mesures d'hygiène, aux vaccins et aux antibiotiques. Pourtant, d'autres pathologies graves, liées à notre environnement et notre alimentation ont émergé sur tous les continents. C'est le cas de l'obésité et du diabète, qui sont des maladies métaboliques concernant la plupart des pays (Roth et al., 2004). La fréquence de ces maladies devrait augmenter partout dans le monde (Zhou et al., 2016). S'y ajoute aujourd'hui la pandémie du coronavirus qui s'inscrit dans une série de zoonoses, des maladies transmissibles de l'animal à l'homme, dont la fréquence a été multipliée par plus de 10 entre 1940 et aujourd'hui. La fréquence des zoonoses devrait aussi augmenter du fait d'une proximité croissante entre des territoires très peuplés et des espaces très «sauvages » constituant des réservoirs de virus dangereux, accentuant ainsi les risques (Destoumieux-Garzón et al., 2018). L'augmentation de la mobilité des personnes, et dans une moindre mesure des produits alimentaires, à toutes les échelles géographiques leur confère un risque pandémique. L'évolution des systèmes alimentaires a eu un poids considérable sur la conjugaison et l'amplification des impacts délétères de ces crises (Tirado et al., 2010), générant alors des effets « domino » et des réactions en chaîne qui complexifient leur analyse.

De ce fait, une approche de ces crises par domaine (agriculture, environnement, alimentation, santé) ou par thème (changement climatique, antibiorésistance, nitrate, biodiversité...), s'est révélée très souvent limitée pour décrire ces dynamiques et identifier des stratégies de transition durables.

Examiner le système alimentaire dans sa globalité et par une approche de «santé globale» prend alors tout son sens. L'approche «une seule santé» (one-health), centrée sur l'importance des relations entre la santé des individus (humains, animaux) et de leur environnement (Mwangi et al., 2016), peut être couplée aux approches de santé environnementale, qui se concentrent sur les maladies dues à l'environnement (Finn et Fallon, 2017), et de santé planétaire, pour prendre en compte les interactions entre états des écosystèmes locaux, état de la planète et santé humaine (Friel et Ford, 2015). On parle alors de «santé globale» (Duru et Therond, 2019).

Notre analyse porte sur les systèmes alimentaires des pays occidentaux en prenant la France comme cas d'étude. Elle est articulée en trois axes :
- L'occupation des sols et les pratiques de gestion des sols, des cultures et de la biomasse (ex. travail du sol, apports d'intrants, récolte/restitution au sol) sont les principaux facteurs ayant un effet sur la santé des agroécosystèmes aux échelles locales, du sol au paysage (Therond et al., 2017). Ils déterminent la structure et le fonctionnement des systèmes sol-plantes et des paysages et, in fine, les services écosystémiques (SE) et la quantité et qualité des «biens» végétaux et animaux produits. Ils influent aussi sur les changements climatiques qui, eux-mêmes, in fine, déterminent la capacité des écosystèmes à fournir des $\mathrm{SE}$ et donc leur santé à l'échelle globale (Runting et al., 2017).

- La gestion des flux de matières (ex. produits ou composés pour l'industrie et les exploitations agricoles...) et d'énergie aux échelles de la ferme, du paysage et jusqu'à la planète (marché international) détermine le degré d'ouverture des cycles biogéochimiques (carbone, azote, phosphore...) et donc les pertes à ces différents niveaux d'organisation (Le Noë et al., 2017), ainsi que les ressources disponibles pour l'agriculture et, in fine, l'offre alimentaire locale.

- L'offre en produits agricoles, leur mode de transformation et les conditions d'accès aux aliments déterminent les choix alimentaires qui impactent la santé humaine, notamment la sensibilité aux infections et le risque de maladies chroniques non transmissibles (Raiten et Aimone, 2017). Le régime alimentaire a aussi un effet sur les émissions des gaz à effet de serre (GES), principalement en fonction de la part de produits animaux (Tilman et Clark, 2014).

Cette représentation du système alimentaire offre un cadre aidant à améliorer la planification, la prise de décision, la gouvernance et la gestion des crises (Ekins et Gupta, 2019). Nous utiliserons cette grille d'analyse pour structurer la littérature scientifique. Dans une première partie nous proposons un diagnostic sur les systèmes alimentaires dominants des pays occidentaux, puis nous analyserons la cohérence entre les politiques publiques et l'engagement des gouvernements pour une transition écologique en prenant la plupart des exemples en France.

\section{Le système alimentaire contribue aux crises environnementales et sanitaires}

\subsection{L'intensification et la spécialisation agricole sont des accélérateurs de l'érosion de la biodiversité et des émissions dans l'eau et l'air}

En vue de répondre aux besoins d'une population croissante et de réaliser des économies d'échelle, l'intensification et la spécialisation des productions agricoles ont entraîné une baisse considérable de la biodiversité cultivée et forestière depuis la fin de la seconde guerre mondiale dans la plupart des régions du monde. Cette érosion a contribué à diminuer la biodiversité dite «associée » à plusieurs échelles (Grab et al., 2018), engendrant alors une réduction des SE essentiels à l'agriculture (par exemple: la régulation des ravageurs ou encore la pollinisation), mais aussi une diminution des services rendus à la société comme l'épuration de l'eau. 
À l'échelle mondiale, la déforestation est à l'origine de $10 \%$ des émissions mondiales des GES. Elle favorise la production d'aliments qui sont loin d'être les meilleurs pour notre santé (par exemple à base d'huile de palme) ou qui rentrent dans la composition des aliments destinés à nourrir les animaux d'élevage (par exemple les tourteaux de soja). Par ailleurs, elle accentue le rapprochement d'une faune sauvage chassée de son habitat naturel vers des animaux domestiques ou les hommes en zones périurbaines, fournissant alors de nouvelles chaînes de transmission et de recomposition possibles à des agents infectieux comme les coronavirus (Afelt et al., 2018).

À l'échelle européenne, en plus d'être à l'origine d'importantes émissions de GES, le labourage des prairies permanentes pour cultiver des plantes annuelles constitue une perte d'habitats pour des espèces qui fournissent une diversité de services écosystémiques pour l'environnement et notre santé. La concentration de l'élevage dans certaines régions entraîne une surabondance d'effluents d'élevage difficiles à gérer sur des surfaces d'épandage réduites, mais aussi de forts apports de fertilisants et d'antibiotiques, entrainant des risques de pollutions (marées vertes) et d'antibiorésistance (Koch et al., 2017).

À l'échelle des territoires, les teneurs en ammoniac de l'air (issu des monogastriques) et d'azote et de phosphore dans les eaux (tous élevages) ont augmenté dans les régions où il y a une forte concentration d'élevages. Parallèlement, les résidus de pesticides dans les sols ou leurs émissions dans l'air ont augmenté dans les zones de grandes cultures. Par ailleurs, l'augmentation importante de la surface moyenne des parcelles, la réduction des infrastructures écologiques (talus, haies) engendre une diminution des régulations biologiques (Sirami et al., 2019). Ces évolutions constituent aussi des facteurs de contamination croissante d'autres habitats par des espèces invasives (plantes, insectes, virus...), ce qui nécessite en retour des besoins plus élevés en intrants de synthèse ( pesticides, apports d'engrais) voire d'eau par l'irrigation.

À l'échelle de la parcelle agricole, la simplification des systèmes de culture (réduction du nombre de cultures en rotation), l'accroissement de l'utilisation des pesticides et le développement trop important du travail du sol entraînent une plus grande dépendance des cultures aux intrants de synthèse exogènes à l'exploitation agricole. La faible teneur des sols cultivés en matières organiques observée ces dernières années, notamment dans les zones de grandes cultures, engendre une augmentation des impacts de l'agriculture sur l'environnement (Garratt et al., 2018).

\subsection{Un régime alimentaire de type occidental n'est bon ni pour la santé, ni pour la planète}

L'industrialisation poussée des procédés de conservation et de transformation des produits agricoles en aliments prêts à consommer a contribué à la sécurité alimentaire, mais elle a drastiquement réduit la diversité de l'offre. Elle a favorisé le développement d'un régime alimentaire de type occidental, cause de dégradation de notre santé (Carrera-Bastos et al., 2011) et de notre environnement (Hyland et al., 2017). Plus particulièrement ce régime alimentaire altère le fonctionnement du microbiote intestinal (Martinez et al., 2017), ce qui est source d'inflammations chroniques à bas bruit (Rinninella et al., 2019) à l'origine de maladies chroniques non transmissibles comme l'obésité et le diabète (Sonnenburg et Sonnenburg, 2019). Ces maladies sont elles-mêmes une porte d'entrée pour d'autres maladies d'origine inflammatoire, comme les maladies cardiovasculaires ou les cancers (Fardet et Boirie, 2013). Les points critiques d'un régime alimentaire occidental standard sont les suivants :

- Une insuffisance de fibres, le «carburant» de notre microbiote intestinal; $90 \%$ de la population française n'atteignent pas le niveau recommandé (PNNS, 2019). Cette insuffisance s'est accentuée depuis le milieu du $\mathrm{XX}^{\mathrm{e}}$ siècle notamment du fait d'une réduction forte de la consommation de légumineuses dont les impacts positifs sur la santé sont reconnus (Dahiya et al., 2017), au profit des protéines animales, et de la mise en marché de farine de blé raffinée.

- Une insuffisance d'oméga 3, 95\% de la population n'atteignant pas le niveau recommandé, alors que ces acides gras jouent un rôle essentiel dans le contrôle des inflammations (Jang et Park, 2020). Cette insuffisance s'est accentuée depuis le milieu du $20^{\mathrm{e}}$ siècle, en partie du fait d'un changement du mode d'alimentation des ruminants et par la suite, de la qualité des produits animaux; l'herbe ayant été remplacée par le maïs et le soja (Duru et Magrini, 2017). Cette évolution jointe à une augmentation de la consommation d'huiles riches en oméga 6 (tournesol) a aussi accru le risque de dysbiose intestinale (Duru, 2019).

- Une consommation excessive de produits «ultra-transformés » à base de produits raffinés, souvent riches en gras et en sucres, contenant des émulsifiants et édulcorants et autres additifs (Miclotte et van de Wiele, 2019), ainsi que la présence de résidus de pesticides même en quantités infimes (Yuan et al., 2019), qui contribuent aussi à l'augmentation des dysbioses intestinales.

- La présence de xénobiotiques dans les aliments, l'eau ou l'air est aussi un facteur de risque pour les maladies métaboliques (Duarte-Hospital et al., 2019).

Ce régime alimentaire contribue aussi à réduire l'efficacité de notre système immunitaire (van den Elsen et al., 2017), nous rendant encore plus vulnérables aux zoonoses. D'une part, le microbiote intestinal ne joue plus son rôle protecteur contre les maladies infectieuses. D'autre part, le diabète, les maladies cardiovasculaires, mais aussi l'obésité et l'hypertension, deviennent des facteurs de développement de formes graves des maladies infectieuses, comme l'a montré l'étude des patients touchés par la Covid-19 en Chine et en Italie (Porcheddu et al., 2020).

Ce type de régime alimentaire n'est pas bon non plus pour la planète. Estimé responsable de 65 à $80 \%$ des émissions de GES de notre système alimentaire (Ademe, 2019), notre consommation en produits animaux est ainsi particulièrement questionnée car la production de protéines animales émet, selon le type d'élevage, entre 5 et 15 fois plus de GES que la production d'une même quantité de protéines végétales (Poore et Nemecek, 2018). Par ailleurs, cette lourde empreinte est renforcée par le fait que les protéines animales sont surconsommées. En France, la consommation moyenne de protéines est de $1,4 \mathrm{~g} / \mathrm{kg}$ de poids corporel et par jour et ce sont 
aux 2/3 des protéines animales (Anses, 2017). C'est à la fois supérieur tant à nos besoins $(0,8 \mathrm{~g} / \mathrm{kg})$ qu'aux recommandations nationales en vigueur ( 1 à $1,2 \mathrm{~g} / \mathrm{kg}$ de poids corporel). Cette surconsommation repose sur la consommation de produits animaux peu chers et encourage alors d'autant plus les formes d'élevage industrielles, très dépendantes de sources exogènes en protéines comme les tourteaux de soja, qui participent à la baisse de la biodiversité du fait de la déforestation pour laisser la place à cette culture (Boerema et al., 2016).

Au-delà d'un impact néfaste sur l'environnement, la proportion de protéines animales dans notre alimentation, d'environ $2 / 3$, est aussi remise en cause pour ses effets sur notre santé. Par exemple, l'excès de consommation de viande rouge et de charcuterie est un facteur de risque pour certains cancers, d'où des recommandations de ne pas dépasser $500 \mathrm{~g}$ de viande rouge et $125 \mathrm{~g}$ de charcuterie par semaine (seuils dépassés respectivement par 32 et $64 \%$ des français) (PNNS, 2019).

\subsection{La globalisation des chaînes de valeur fragilise la sécurité alimentaire}

Tout comme l'industrie de biens manufacturés, la production, la transformation et la distribution des aliments impliquent de nombreuses entreprises, souvent localisées dans plusieurs pays. La production au sein de ces chaînes de valeur se fait largement à flux tendus, pour minimiser les coûts de stockage à chaque point de la chaîne. Ces dynamiques permettent avant tout aux pays de gagner en compétitivité et de réaliser des économies d'échelle, que ce soit pour la fourniture des aliments pour les animaux d'élevage ou de certaines familles de produits pour l'alimentation humaine. Elles présentent cependant deux inconvénients majeurs. Si ces modes de distribution permettent de bénéficier directement de gains liés à la spécialisation des entreprises et des pays, ils rendent également l'économie particulièrement vulnérable à des chocs locaux, ou bien à un choc plus global comme celui récent de la pandémie de la Covid-19. En effet, une perturbation de la production dans un pays dominant (cas du soja) ou du transport des produits peut notamment paralyser l'ensemble de la chaîne de valeur et accentuer localement la faible résilience d'autres territoires aux crises environnementales et sanitaires.

Le commerce international d'aliments, intra ou extra Europe, contribue à accentuer les déséquilibres des cycles naturels des éléments chimiques comme l'azote et le phosphore dans certaines régions du monde. Par exemple, de 1961 à 2010, la quantité de produits contenant de l'azote échangés entre les pays a presque été multipliée par 8 et représente désormais un tiers de l'azote contenu dans la production agricole mondiale totale (Therond et al., 2017). À ce titre, les élevages intensifs de volailles ou de porcs où sont concentrés sur de petits espaces un nombre important d'animaux font problème: ils reposent sur des importations massives d'aliments riches en azote et les surfaces d'épandage à proximité des bâtiments ne sont pas toujours suffisantes pour recycler au mieux les déjections animales. En outre, la concentration massive d'animaux est susceptible d'accroître les risques sanitaires et l'usage des médicaments pour les gérer.
Les choix de consommation renforcent aussi cette mondialisation. C'est le cas bien sûr des achats de produits frais (légumes) hors saison. Mais il s'agit aussi des types de viande. Ainsi la restauration hors domicile provient essentiellement de viande importée, correspondant aux quartiers avant de vaches laitières réformées. Au niveau européen, la forte spécialisation et intensification des volailles entre poules pondeuses et poulets de chair pour répondre à une demande croissante, génère des poules de réforme sans grande valeur. L'augmentation importante de la consommation de blancs de poulet, notamment en restauration collective, déclasse les cuisses. Aussi les poules de réforme et les cuisses sont vendues à bas prix en Afrique, déstabilisant les filières de volailles dans les pays où ces produits sont importés.

\section{Transformer le système alimentaire pour l'environnement et la santé}

\subsection{Trois axes pour une transition forte du champ à l'assiette}

\subsubsection{Favoriser des modes de production s'inscrivant dans une démarche agroécologique}

Promouvoir une agriculture qui offre une diversité de produits de qualité, qui stocke du carbone dans les sols et fournit des produits animaux basés sur la diversité des plantes, des paysages et des organismes du sol, ainsi que le renforcement des synergies entre cultures et élevage (Garrett et al., 2020), suppose un changement de paradigme majeur, plaçant la santé du sol (Geisen et al., 2019) au cœur de la transition, et l'élevage comme une activité de "support» (Eldridge et Delgado-Baquerizo, 2017). Une agriculture basée sur la santé du sol (Wang et Li, 2019) contribue à la santé des plantes, des animaux, de la planète et à la santé humaine. Elle ne repose pas uniquement sur l'adoption de technologies permettant de réduire les impacts dans tel ou tel domaine en appliquant «le bon produit, au bon moment, au bon endroit et à la bonne dose» (Therond et al., 2017). Cette orientation nécessite aussi l'implication de l'ensemble des organisations professionnelles agricoles en charge de la collecte, du stockage, et de la traçabilité de ces produits pour le consommateur.

Selon la stratégie nationale bas carbone, l'agriculture française doit aussi stocker du carbone dans le sol pour compenser les émissions résiduelles des autres secteurs de l'économie et fournir des produits biosourcés (énergie, matériaux) (Poeplau et Don, 2015). Pour fixer le cap, rappelons que l'agriculture hors prairies déstocke du carbone. Une étude récente montre qu'en France, les sols cultivés déstockent en moyenne $170 \mathrm{~kg}$ de $\mathrm{C} / \mathrm{ha} / \mathrm{an}$ (Pellerin et al., 2019). La généralisation des cultures intermédiaires semées entre deux cultures principales, jointe à la réduction du travail du sol, sont les principaux leviers (Büchi et al., 2018) pour inverser la tendance, ce qui permettrait d'augmenter la teneur en matières organiques et de renforcer l'activité biologique et l'accès à plus de ressources pour les plantes (nutriments, eau) de façon à réduire les apports d'intrants de synthèse. La fabrication des produits biosourcés ne doit cependant pas entrer en compétition avec l'alimentation humaine, ce qui suppose de ne pas développer des cultures dédiées à cela 
(biocarburants de première génération). Par contre, la méthanisation des déjections animales ou de cultures intermédiaires avec retour des digestats au champ permet de récupérer de l'énergie sans altérer le cycle des nutriments (Szerencsits et al., 2016).

Pour les cultures de base (céréales, oléo-protéagineux), l'enjeu est de réintroduire de la biodiversité en termes de diversité de cultures par des cultures associées et intercultures (Wezel et al., 2014) et des infrastructures écologiques (haies, bandes enherbées...) pour soutenir les pollinisateurs et les ennemis naturels tels que les prédateurs et les parasitoïdes, même si les pratiques agricoles restent intensives sur les terres cultivées (Redhead et al., 2020). Les légumineuses en cultures pures, associées ou en intercultures, ont un rôle essentiel à jouer (Schneider et Huyghe, 2015). De plus en plus de recherches se référant à la santé du sol invitent à considérer ces différentes formes de biodiversité et la matière organique du sol afin de modifier la rhizosphère (les racines des plantes et le microbiome associé) ainsi que le microbiote du sol. Ces composants interagissent de multiples façons et remplissent collectivement de multiples fonctions, telles que l'amélioration de la disponibilité des nutriments, la prévention des ravageurs et des maladies, le stockage du carbone et l'amélioration de la structure du sol et de la capacité de rétention d'eau (Zhang et al., 2020). Une des controverses porte sur les formes d'agriculture biodiversifiée à promouvoir. La biodiversité dans le sol est favorisée par l'absence d'utilisation de pesticides de synthèse et le non-labour, voire l'absence de travail du sol. L'agriculture biologique mobilise le premier levier mais pas le second; pour l'agriculture de conservation des sols, c'est l'inverse du fait de l'utilisation quasi systématique d'herbicides, si bien qu'il est à ce jour difficile de concilier les deux leviers (Duru et al., 2020).

L'élevage, compte tenu de ses forts impacts sur l'environnement, doit être redimensionné à la baisse et réorienté (Weind et al., 2020). Pour cela, il doit être conçu comme un moyen d'utiliser des ressources n'entrant pas en compétition avec l'alimentation humaine (prairies permanentes pour les ruminants, déchets de l'agro-alimentaire pour les monogastriques) ou comme un coproduit des cultures (pâturage de cultures intermédiaires, d'inter-rangs enherbés de cultures pérennes), ou encore comme un moyen d'apporter des nutriments spécifiques comme les acides gras indispensables qu'on ne trouve pas dans les produits végétaux comme les oméga 3 à très longue chaîne (Duru et Magrini, 2016). Cette option limite fortement l'utilisation des céréales en production animale. Ce changement de paradigme doit en outre être concomitant à la déconcentration de l'élevage à l'échelle des fermes et des régions de façon à réduire les impacts environnementaux négatif sur l'air et le sol, améliorer le bien-être animal (Weishaupt et al., 2020) mais aussi réduire les risques épidémiques (Pecl et al., 2017). Impulser le développement de modes de production agroécologiques permet indirectement de réduire le risque de zoonoses : (i) en diminuant la pression sur les ressources importées (arrêt des importations de soja, moins de pétrole ou de gaz); (ii) en réduisant les formes d'élevage les plus intensives en termes de consommation d'antibiotiques et d'émissions dans l'environnement; (iii) en contribuant à nous offrir une alimentation plus diversifiée et plus saine. Une des controverses porte sur le type de production à réduire: les ruminants du fait des fortes émissions de GES et de leur faible efficience protéique mais pouvant valoriser des prairies, ou les monogastriques, meilleurs sur les deux premiers points, mais entrant bien plus en compétition avec l'alimentation humaine (Peyraud, 2017).

\subsubsection{Rééquilibrer notre alimentation vers plus de végétal, de diversité et d'aliments moins transformés}

Les choix de consommation soutiennent la pérennité des modes de production, transformation et distribution des aliments. C'est par exemple le cas pour des légumes frais achetés hors saison qui favorisent l'agriculture sous serre chauffée ou les importations sur de longues distances, ou pour certains plats en restauration hors domicile comme les burgers qui privilégient des parties avant de viande bovine, largement importées.

Promouvoir une alimentation préservant le microbiote intestinal suppose de passer d'une vision analytique des aliments par famille (viande, légumes...) ou par nutriments ( lipides, glucides, fer...), à une vision élargie reposant sur la capacité des aliments et de leur association à bien "nourrir» notre corps et donc notre microbiote (van de Guchte et al., 2018). Pour cela, il s'agit d'avoir un apport en fibres, en oméga 3 et antioxydants à la hauteur des recommandations et des apports modérés de protéines animales (viande) comme indiqué en 2.2. Prioriser la consommation de légumineuses riches en fibres (Margier et al., 2018) et de produits animaux à teneur élevée en oméga 3 (Mourot et Tonnac, 2015) est incontournable pour atteindre les apports nutritionnels recommandés. Il en est de même pour la réduction des aliments à base de produits raffinés, contenant des additifs tels que les édulcorants et émulsifiants, ainsi que des contaminants. Une telle vision holistique passe ainsi par une forte réduction de consommation de produits ultra-transformés (Miclotte et van de Wiele, 2019), presque toujours associée à une faible diversité de matières premières qui réduit la diversité du microbiote intestinal.

Promouvoir une alimentation préservant l'environnement passe essentiellement par le choix de produits ne contribuant pas à la déforestation en zones tropicales et surtout au changement climatique. Cela implique que les modes de production, ou encore le mode d'alimentation des animaux, doivent systématiquement être tracés et renseignés pour le consommateur afin qu'il puisse faire un choix en toute connaissance. Pour fixer le cap, rappelons que l'empreinte carbone actuelle d'un français est de 11,2 t équivalent $\mathrm{CO}_{2}$ par personne et par an; elle doit être ramenée entre 1,6 et $2,8 \mathrm{teq} \mathrm{CO}_{2}$ pour limiter le réchauffement à $2^{\circ} \mathrm{C}$ en 2050. L'empreinte actuelle de notre alimentation, de 2,4 teq $\mathrm{CO}_{2}$ dont $1,6 \mathrm{t}$ eq $\mathrm{CO}_{2}$ pour la production agricole, est donc proche de ce quota. Les experts s'accordent pour dire que pour décarboner l'économie, il faut diviser l'empreinte de notre alimentation par 2 ( $-46 \%$ entre 2015 et 2050$)$ (Ministère de la transition écologique et solidaire, 2020).

Pour donner un ordre de grandeur des recommandations de baisse de consommation en produits animaux envisagée, le plus récent Plan national nutrition santé (PNNS) propose un apport équilibré entre protéines végétales et animales, permettant de réduire de $16 \%$ les émissions de GES de l'alimentation. Un régime de type méditerranéen est encore meilleur pour l'environnement et la santé (Dernini et al., 
2017). Avec $60 \mathrm{~g}$ de protéines/jour dont $2 / 3$ de protéines végétales, il permettrait de réduire de près de moitié les émissions de GES de notre alimentation. Le niveau de réduction fait cependant débat compte tenu des autres nutriments à considérer (vitamine B12...) et du fait d'un meilleur équilibre entre acides aminés pour la viande en comparaison des protéines végétales (Bohrer, 2017).

En résumé, manger «plus végétal», plus varié et moins transformé limite les risques pour notre santé (Sommer et al., 2017; Fardet et Rock, 2020) et permet de réduire les émissions de GES. Ces régimes alimentaires peuvent aussi indirectement contribuer à réduire le risque d'issues dramatiques aux pandémies en renforçant notre système immunitaire à tous les âges de la vie (Martinez, 2017).

\subsubsection{Revoir les chaînes de valeur pour garantir une alimentation saine et durable à tous}

La forte dépendance aux échanges et aux chaînes de valeur mondialisées, associée à une main-d'œuvre précarisée, et une absence notable de diversité de productions et de débouchés, doivent être réduites. Plusieurs changements majeurs doivent être entrepris de manière concertée entre tous les acteurs du système.

Les accords internationaux de libre échange favorisent toujours la spécialisation des régions et des continents. Ils permettent souvent des réductions de prix via des économies d'échelle et d'agglomération. Mais leur évaluation ne prend pas en compte la diversité des critères de performances que nous avons considérés. C'est pourquoi ils sont généralement source de dégradation environnementale et sociale. À titre d'exemple, les produits les plus mondialisés dans certains secteurs (fromage, jambon, vin et pain) sont mal classés pour un ensemble de critères (environnement, social, santé, éthique), mais ils présentent deux atouts : prix bas et émissions de GES plus faibles (Schmitt et al., 2017). Ils ne permettent cependant pas de freiner la destruction d'habitats avec les conséquences sur la biodiversité, et ils sont aussi un frein à la diversification des cultures.

Des systèmes alimentaires plus déconcentrés, encourageant la production et la consommation locales et la fourniture de services écosystémiques sont donc à privilégier (Toth et al., 2016). Réduire la dépendance des pays européens aux importations de soja et d'huile de palme est aussi un moyen d'enrayer le déclin de l'agriculture familiale vivrière face à la prédominance d'une agriculture « industrialisée » dans les pays exportateurs (Oliveira et Hecht, 2016).

Raccourcir les chaînes d'approvisionnement peut contribuer à réduire les émissions dues aux transports. Pour fixer le cap, rappelons que la consommation d'énergie pour le système alimentaire français (Barbier et al., 2019) est d'environ 31,6 Mtep dont 8,4 pour l'agriculture ; l'essentiel étant dû aux transports $(9,7)$ puis aux industries agro-alimentaires (4,9 Mtep); le reste provenant du commerce et de la consommation à domicile. Réduire certains circuits d'approvisionnement est donc un levier pour réduire l'empreinte carbone.

Réduire la production et la distribution de produits ultratransformés et/ou trop riches en sucres et graisses nécessite l'adaptation de l'industrie agro-alimentaire, pour mieux renseigner la valeur santé des aliments ainsi que leur impact environnemental. Des systèmes de classification des aliments basés sur des connaissances scientifiques peuvent encourager les changements chez les industriels et les consommateurs. Un affichage nutritionnel comme le Nutriscore ou couplant nutrition et degré de transformation (méthode SIGA) (Davidou et al., 2020) est pertinent. Il serait à compléter par un affichage environnemental.

Diversifier les cultures et raccourcir les circuits d'approvisionnement, mettre sur le marché moins de produits ultratransformés suppose de réorganiser la collecte, le stockage, et aussi renforcer la traçabilité de ces produits pour le consommateur (Ponisio et Ehrlich, 2016), et donc d'impliquer les acteurs de l'agrofourniture, de la transformation et de la distribution pour coordonner les changements à venir. Des modes de transformation et distribution ancrés dans les territoires et cohérents avec le rééquilibrage de notre régime alimentaire permettent d'assurer la conception de filières de production alimentaire locales. Cela permet de rapprocher les consommateurs et les producteurs, ce qui contribue à la transparence et à la confiance (Bowen et Mutersbaugh, 2014).

Un point de controverse concerne les circuits courts. D'une part, il n'est pas évident que cela puisse toujours réduire les impacts environnementaux si les producteurs et les consommateurs font plus de trajets routiers. D'autre part, c'est impossible à mettre en œuvre pour les métropoles. Pour cette dernière raison, il est admis que ces systèmes sont à privilégier pour les produits frais tels les fruits et légumes (Benis et Ferrao, 2017).

\subsection{Une réorientation majeure : la territorialisation des systèmes alimentaires}

L'analyse de la littérature dans les trois domaines (agriculture, alimentation, chaîne d'approvisionnement) montre, au-delà des controverses, la nécessité de renforcer les interactions entre acteurs au sein d'un territoire, que ce soit pour développer des interactions biologiques (associations de cultures, mosaïques paysagères), des modes d'organisation (association culture-élevage au niveau de l'exploitation agricole ou du territoire) ou pour construire plus de confiance entre les acteurs du système alimentaire. Renforcer l'ensemble de ces interactions passe par le développement de systèmes alimentaires territorialisés. Une telle orientation permet de combiner des évolutions des modes de production, de transformation, de distribution et de consommation diversifiés et raisonnés, minimisant les effets domino des impacts des crises environnementales et sanitaires en améliorant la résilience environnementale et sanitaire des territoires (Marsden et al., 2018). Trois principes gouvernent de ce fait cette réorientation stratégique majeure à conduire: l'autonomie, la proximité et la solidarité (Rastoin et Ghersi, 2010).

- L'autonomie correspond à un objectif d'accroissement de l'autosuffisance en intrants pour l'agriculture et en denrées alimentaires de base. Ce principe limite la longueur et la complexité des filières agro-industrielles, aujourd'hui de plus en plus mondialisées et des dispositifs logistiques et de gouvernance les accompagnant.

- La proximité suggère le développement de synergies entre activités agricoles (culture, élevage, forêt). Il s'agit aussi de renforcer la valorisation locale des matières premières 
agricoles, par exemple pour l'engraissement des animaux, la fabrication d'aliments peu transformés, par la formation de réseaux contractuels favorables au partage de la valeur.

- La solidarité passe par des statuts d'entreprise intégrant la responsabilité sociale et environnementale, des formes coopératives d'organisation des filières et une mutualisation des ressources.

De tels systèmes alimentaires peuvent se concevoir à différentes échelles, depuis les régions jusqu'aux États, pour peu qu'une gouvernance soit définie sur la base des principes qui les régissent. Cependant, la réussite de leur mise en œuvre suppose de créer des conditions propices au niveau des politiques publiques. Un récit puissant, rassembleur, coconstruit et partagé, peut servir de cadrage à la mise en œuvre d'une telle transition qui allie autant des changements de modes de production, transformation, distribution et de consommation des aliments (Borsellino et al., 2020). C'est aussi une condition pour prendre en compte les différentes dimensions de ce changement : l'environnement, la santé, mais aussi l'économie et le social, non considérés dans cette note. Ils doivent être élaborés et partagés aux différents niveaux des politiques publiques aux échelles mondiales (révision des accords de libre-échange), de l'Europe (la PAC), de la France et des régions, de façon à veiller aux cohérences et tenir compte des spécificités des territoires.

L'émergence progressive de Projets alimentaires de territoire (PAT) dans les territoires français constitue une première étape d'amorce pour la concertation et le dialogue à mener entre les acteurs du changement (agriculteurs, consommateurs, industriels, grands distributeurs, commerçants indépendants, restauration hors domicile, consommateurs, etc.) (Assemblée nationale, 2018). À ce jour, une large majorité de projets, déposés comme lauréats, demeurent centrés sur un renforcement de l'approvisionnement des cantines scolaires en produits locaux (ou AB). Depuis 2016, il s'agit aussi de projets monothématiques portant sur les axes «Ancrage territorial et approvisionnement de proximité » et «Gaspillage alimentaire» du plan national de l'alimentation (Wallet, com. pers.). L'enjeu n'est pas de rendre les territoires autonomes pour tous les intrants et tous les aliments, mais d'augmenter la production et la consommation de ceux qui peuvent à la fois rendre l'agriculture plus résiliente (légumineuses par exemple), ou qui sont clefs pour l'alimentation (légumes par exemples), ou qui sont coûteux à transporter du fait de leurs caractéristiques (fruits et légumes).

\section{Conclusion: biodiversité et sobriété pour faire face aux grands défis}

Notre analyse, circonscrite au niveau du système alimentaire, a montré que la dimension écologique des récentes crises environnementales et sanitaires provient du déclin voire de l'effondrement de la biodiversité dans des domaines très différents (écosystèmes naturels et cultivés, intestin) et dans des espaces éloignés les uns des autres (en France, dans les pays fournisseurs de produits de base). Cette baisse de diversité se traduit par une réduction de services écosystémiques fournit par les écosystèmes, qu'il s'agisse de notre intestin pour fabriquer des molécules anti-inflammatoires, du rumen de la vache, du système sol-plante pour accéder à des ressources peu mobilisables ou protéger les plantes des agressions des perturbations biotiques et abiotiques, d'écosystèmes naturels (forêts, zones humides...) pour contenir des bio-agresseurs.

L'approche systémique de «santé globale» que nous avons proposée a mis en évidence l'interdépendance de l'état de santé des hommes, des animaux et des écosystèmes dans lesquels ils évoluent. Les analyses fournissent des pistes et des arguments pour éclairer les politiques publiques qui doivent être bien plus préventives et systémiques qu'elles ne le sont. Nous avons montré qu'il faut coordonner des changements dans les modes de production, de transformation, de distribution et de consommation des aliments : réorienter l'élevage, abaisser le degré de transformation des aliments, diversifier les modes de distribution et végétaliser notre assiette. Ces changements participent à la territorialisation du système alimentaire.

Pour aller plus loin, il importe de concevoir des scénarios où les choix sont quantifiés de façon à vérifier la cohérence des options prises et la diversité des impacts. De tels exercices ont été faits au niveau français (Solagro, 2014), européen (Poux et Aubert, 2018) et mondial (de Lattre-Gasquet et al., 2017). Leur utilisation doit être une aide pour bâtir un système alimentaire à même de satisfaire de légitimes attentes sanitaires, environnementales, sociétales et économiques (Duru et Caron, 2020)

Pour définir les arbitrages à réaliser, les études complémentaires à conduire sont complexes car il y a une forte diachronie dans le changement des pratiques agricoles et alimentaires et leurs effets sur l'environnement ou la santé. En outre, les domaines du vivant étant interconnectés, notamment via les microorganismes (van Bruggen et al., 2018 ; Flandroy et al., 2018), un changement peut entraîner des effets dominos lorsqu'un maillon de la chaîne est fragilisé. D'autre part, dans chacun des domaines, les politiques et pratiques choisies ne font parfois qu'accentuer le déclin de la biodiversité, engendrant ainsi des cercles vicieux du fait de la réduction de services fournis par les écosystèmes (Zimmerer et al., 2019). Concevoir l'agriculture, l'alimentation et plus généralement la gestion de la biodiversité selon les critères de performances de l'industrie (économie d'échelle, gestion en flux tendu, stratégie de court terme...) en ignorant le vivant est un contresens. On en oublie que le vivant rend des services dans de nombreux domaines (sol, agriculture, écosystèmes naturels et cultivés, notre intestin...) (Aronson et al., 2016; Zhu et al., 2019). La biodiversité offre la possibilité de développer des synergies que ce soit au niveau de l'intestin, du sol, des paysages, des continents. Ses effets seront d'autant plus marqués que les modes de production, transformation, distribution et d'alimentation seront sobres pour la consommation d'énergie et les émissions de GES, en priorisant les besoins en biens et services les plus essentiels.

\section{Références}

Ademe. 2019. www.ademe.fr/bilan-carbone-lalimentation-france. Afelt A, Frutos R, Devaux C. 2018. Bats, coronaviruses, and deforestation: Toward the emergence of novel infectious diseases? 
Frontiers in Microbiology 9(APR): 1-5. DOI: 10.3389/ fmicb.2018.00702.

Anses. 2017. www.anses.fr/fr/system/files/NUT2014SA0234Ra.pdf. Assemblée nationale. 2018. www.assemblee-nationale.fr/dyn/15/ rapports/cealimindu/115b1266-ti_rapport-enquete\#_ Toc256000105.

Aronson JC, Blatt CM, Aronson TB. 2016. Restoring ecosystem health to improve human health and well-being. Ecological and Society 21(4). DOI: 10.5751/ES-08974-210439.

Barbier C, Couturier C, Pourouchottamin PR, Cayla JMR, Silvestre M, Billen G. 2019. Le bilan énergétique et carbone de l'alimentation en France de la production à la consommation. Club Ingénierie Prospective Energie et Environnement. Paris : IDDRI, $24 \mathrm{p}$.

Benis K, Ferrao P. 2017. Potential mitigation of the environmental impacts of food systems through urban and peri-urban agriculture: a life cycle assessment approach. Journal of Cleaner Production 140: 784-795. DOI: 10.1016/j.jclepro.2016.05.176.

Boerema A, Peeters A, Swolfs S, Vandevenne F, Jacobs S, Staes J, et al. 2016. Soybean trade: Balancing environmental and socioeconomic impacts of an intercontinental market. PLOS ONE 11(5): 1-13. DOI: 10.1371/journal.pone.0155222.

Bohrer BM. 2017. Nutrient density and nutritional value of meat products and non-meat foods high in protein. Trends in Food Science \& Technology 65: 103-112. DOI: 10.1016/j. tifs.2017.04.016.

Borsellino V, Schimmenti E, El Bilali H. 2020. Agri-Food Markets towards Sustainable Patterns. Sustainability 12(6): 2193. DOI: 10.3390/su12062193.

Bowen S, Mutersbaugh T. 2014. Local or localized? Exploring the contributions of Franco-Mediterranean agrifood theory to alternative food research. Agriculture and Human Values 31(2): 201-213. DOI: $10.1007 / \mathrm{s} 10460-013-9461-7$.

Büchi L. Wendling M, Amossé C, Necpalova M, Charles R. 2018. Importance of cover crops in alleviating negative effects of reduced soil tillage and promoting soil fertility in a winter wheat cropping system. Agriculture, Ecosystems \& Environment 256: 92-104. DOI: 10.1016/j.agee.2018.01.005.

Carrera-Bastos P, Fontes M, O'Keefe J, Lindeberg S, Cordain L. 2011. The western diet and lifestyle and diseases of civilization. Research Reports in Clinical Cardiology 15. DOI: 10.2147/RRCC. S16919.

Dahiya DK, Puniya M, Shandilya UK, Dhewa T, Kumar N, Shukla P. 2017. Gut microbiota modulation and its relationship with obesity using prebiotic fibers and probiotics: A review. Frontiers in Microbiology 8(APR). DOI: 10.3389/fmicb.2017.00563.

Davidou S, Christodoulou A, Fardet A, Frank K. 2020. The holisticoreductionist Siga classification according to the degree of food processing: an evaluation of ultra-processed foods in French supermarkets. Food \& Function 11(3): 2026-2039. DOI: 10.1039/ C9FO02271F.

de Lattre-Gasquet M, Mora O, Le Mouël C, Dumas P, Brunelle T. 2017. Que nous apprend le scénario «Des usages des terres pour une alimentation de qualité et une nutrition saine "de la prospective" Agrimonde-Terra»? In: Rastoin J-L, Ferault C, eds. La sécurité alimentaire mondiale. État des lieux et prospectives. Paris: L'Harmattan, pp. 131-162.

Dernini S, Berry EM, Serra-Majem L, La Vecchia C, Capone R, Medina FX, et al. 2017. Med Diet 4.0: The Mediterranean diet with four sustainable benefits. Public Health Nutrition 20(7): 13221330. DOI: $10.1017 / \mathrm{S} 1368980016003177$.

Destoumieux-Garzón D, Mavingui P, Boetsch G, Boissier J, Darriet F, Duboz P, et al. 2018. The one health concept: 10 years old and a long road ahead. Frontiers in Veterinary Science 5(FEB): 1-13. DOI: 10.3389 /fvets.2018.00014.

Duarte-Hospital C, Huc L, Bortoli S, Coumoul X. 2019. Les xénobiotiques, quel impact sur les maladies métaboliques ? Cahiers de Nutrition et de Diététique 54(5): 286-293. DOI: 10.1016/j. cnd.2019.07.002

Duru M. 2019. Agri-food choices for health: the case of fatty acids. OCL-Oleagineux Corps Gras Lipides 26(44): 11. DOI: 10.1051/ ocl/2019038.

Duru M, Magrini M. 2016. Consommer des produits dont les animaux ont été alimentés à l'herbe est-il suffisant pour équilibrer notre alimentation en acides gras poly-insaturés ? Fourrages 301-312.

Duru M, Magrini M-B. 2017. Composition en acides gras polyinsaturés de notre assiette et utilisation des matières premières agricoles en France: une amélioration lente, mais insuffisante. OCL-Oleagineux Corps Gras Lipides 24(2): A201, 10 p. DOI: 10.1051/ocl/2017007.

Duru M, Therond O. 2019. La «santé unique» pour reconnecter agriculture, environnement et alimentation. Revue Regards RO8.

Duru M, Caron P. 2020. Pourquoi notre santé et celle de la planète se jouent dans nos assiettes. theconversation.com/pourquoi-notresante-et-celle-de-la-planete-se-jouent-dans-nos-assiettes-140500.

Duru M, Sarthou JP, Therond O. 2020. Analyse de modèles d'agriculture au prisme de la «santé unique». Revue Regards RO9.

Ekins P, Gupta J. 2019. Perspective: a healthy planet for healthy people. Global Sustainability 2(e20): 1-9. DOI: 10.1017/ sus.2019.17.

Eldridge DJ, Delgado-Baquerizo M. 2017. Continental-scale impacts of livestock grazing on ecosystem supporting and regulating services. Land Degradation \& Development 28(4): 1473-1481. DOI: $10.1002 / 1 \mathrm{dr} .2668$.

Fardet A, Boirie Y. 2013. Associations between diet-related diseases and impaired physiological mechanisms: A holistic approach based on meta-analyses to identify targets for preventive nutrition. Nutrition Reviews 71(10): 643-656. DOI: 10.1111/nure.12052.

Fardet A, Rock E. 2020. How to protect both health and food system sustainability? A holistic "global health"-based approach via the 3V rule proposal. Public Health Nutrition 23(16): 3028-3044. DOI: $10.1017 / \mathrm{S} 136898002000227 \mathrm{X}$.

Finn S, Fallon LO. 2017. Commentary the emergence of environmental health literacy-From its roots to its future potential. Environmental Health Perspectives 125(4): 495-501. DOI: 10.1289/ehp.1409373.

Flandroy L, Poutahidis T, Berg G, Clarke G, Dao MC, Decaestecker E, et al. 2018. The impact of human activities and lifestyles on the interlinked microbiota and health of humans and of ecosystems. Science of the Total Environment 627: 1018-1038. DOI: 10.1016/j. scitotenv.2018.01.288.

Friel S, Ford L. 2015. Systems, food security and human health. Food Security 7(2): 437-451. DOI: 10.1007/s12571-015-0433-1.

Garratt MPD, Bommarco R, Kleijn D, Martin E, Mortimer SR, Taka V, et al. 2018. Enhancing Soil Organic Matter as a Route to the Ecological Intensification of European Arable Systems. Ecosystems. DOI: 10.1007/s10021-018-0228-2.

Garrett RD, Ryschawy J, Bell LW, Cortner O, Ferreira J, Garik AV, et al. 2020. Drivers of decoupling and recoupling of crop and livestock systems at farm and territorial scales. Ecology and Society 25(1): 24. DOI: 10.5751/ES-11412-250124.

Geisen S, Wall DH, van der Putten WH. 2019. Review Challenges and Opportunities for Soil Biodiversity in the Anthropocene. Current Biology 29(19): PR1036-R1044. DOI: 10.1016/j.cub.2019.08.007.

Gordon LJ, Bignet V, Crona B, Henriksson PJG, Van Holt T, Jonell M, et al. 2017. Rewiring food systems to enhance human health and 
biosphere stewardship. Environmental Research Letters 12(10). DOI: 10.1088/1748-9326/aa81dc.

Grab H, Danforth B, Poveda K, Loeb G. 2018. Landscape simplification reduces classical biological control and crop yield. Ecological Applications 0(0): 1-8.

Hyland J, Henchion M, McCarthy M, McCarthy SN. 2017. The role of meat in strategies to achieve a sustainable diet lower in greenhouse gas emissions: A review. Meat Science 132: 189-195. DOI: 10.1016/j.meatsci.2017.04.014.

Ipes Food. 2019. www.ipes-food.org.

Jang H, Park K. 2020. Omega-3 and omega-6 polyunsaturated fatty acids and metabolic syndrome: A systematic review and metaanalysis. Clinical Nutrition 39(3): 765-773. DOI: 10.1016/j. clnu.2019.03.032.

Koch BJ, Hungate BA, Price LB. 2017. Food-animal production and the spread of antibiotic resistance: the role of ecology. Frontiers in Ecology and the Environment 15(6): 309-318. DOI: 10.1002/ fee. 1505 .

Le Noë J,Billen G, Garnier J. 2017. How the structure of agro-food systems shapes nitrogen, phosphorus, and carbon fluxes: The generalized representation of agro-food system applied at the regional scale in France. Science of The Total Environment 586: 42-55. DOI: 10.1016/j.scitotenv.2017.02.040.

Margier M, Georgé S, Hafnaoui N, Remond D, Nowicki M, Du Chaffaut L, et al. 2018. Nutritional composition and bioactive content of legumes: Characterization of pulses frequently consumed in France and effect of the cooking method. Nutrients 10(11) : 1-12. DOI: 10.3390/nu10111668.

Marsden T, Hebinck P, Mathijs E. 2018. Re-building food systems: embedding assemblages, infrastructures and reflexive governance for food systems transformations in Europe. Food Security 10(6): 1301-1309. DOI: 10.1007/s12571-018-0870-8.

Martinez GP. 2017. Gut Microbiota, a Key Factor Relating Diet and Inflammation with the Progression of Cognitive Impairment in Older People. Journal of Nutritional Health \& Food Engineering 6 (2): 1-7. DOI: 10.15406/jnhfe.2017.06.00192.

Martinez KB, Leone V, Chang EB. 2017. Western diets, gut dysbiosis, and metabolic diseases: Are they linked? Gut Microbes 8(2): 130142. DOI: 10.1080/19490976.2016.1270811.

Miclotte L, Van de Wiele T. 2019. Food processing, gut microbiota and the globesity problem. Critical Reviews in Food Science and Nutrition 60(11): 1769-1782. DOI: 10.1080/ 10408398.2019.1596878.

Ministère de la transition écologique et solidaire. 2020. La transition écologique et solidaire vers la neutralité carbone. www.ecologiquesolidaire.gouv.fr/sites/default/files/SNBC- $2 \% 20$ synthe $\%$ CC $\%$ 80se\%20VF.pdf.

Mourot J, Tonnac A de. 2015. The Bleu Blanc Cœur path: impacts on animal products and human health. OCL 22(6) D610. DOI: 10.1051/ocl/2015051.

Mwangi W, de Figueiredo P, Criscitiello MF. 2016. One Health: Addressing Global Challenges at the Nexus of Human, Animal, and Environmental Health. PLoS Pathogens 12(9): 1-8. DOI: 10.1371/journal.ppat.1005731.

Oliveira G, Hecht S. 2016. Sacred groves, sacrifice zones and soy production: globalization, intensification and neo-nature in South America. Journal of Peasant Studies 43(2): 251-285. DOI: 10.1080/03066150.2016.1146705.

Pecl GT, Araújo MB, Bell JD, Blanchard J, Bonebrake TC, Chen C, et al. 2017. Biodiversity redistribution under climate change: Impacts on ecosystems and human well-being. Science 355(6332). DOI: $10.1126 /$ science.aai9214.
Pellerin S, Bamière L (pilotes scientifiques), et al. 2019. Stocker du carbone dans les sols français. Quel potentiel au regard de l'objectif 4 pour 1000 et à quel coût? Synthèse du rapport d'étude. France : INRA, $114 \mathrm{p}$.

Peyraud MJ. 2017. L'élevage contribue à la production durable de protéines. Demeter 363-388.

PNNS. 2019. www.mangerbouger.fr/pro/sante/alimentation-19/nou velles-recommandations-adultes/1111-test-sous-rubrique/ rubrique-2-article.html.

Poeplau C, Don A. 2015. Carbon sequestration in agricultural soils via cultivation of cover crops - A meta-analysis. Agriculture, Ecosystems \& Environment 200: 33-41. DOI: $10.1016 / \mathrm{j}$. agee.2014.10.024.

Ponisio LC, Ehrlich PR. 2016. Diversification, yield and a new agricultural revolution: Problems and prospects. Sustainability (Switzerland) 8(11): 1-15. DOI: 10.3390/su8111118.

Poore J, Nemecek T. 2018. Reducing food's environmental impacts through producers and consumers. Science 992(6392): 987-992. DOI: $10.1126 /$ science.aaq0216.

Porcheddu R, Serra C, Kelvin D, Kelvin N, Rubino S. 2020. Similarity in Case Fatality Rates (CFR) of COVID-19/SARSCOV-2 in Italy and China. The Journal of Infection in Developing Countries 14(02): 125-128. DOI: 10.3855/jidc.12600.

Poux X, Aubert PM. 2018. An agroecological Europe in 2050: multifunctional agriculture for healthy eating. Findings from the Ten Years For Agroecology (TYFA) modelling exercise, IddriAScA, Study, (09/18).

Raiten DJ, Aimone AM. 2017. The intersection of climate/ environment, food, nutrition and health: crisis and opportunity. Current Opinion in Biotechnology 44: 52-62. DOI: 10.1016/j. copbio.2016.10.006.

Rastoin JL, Ghersi G. 2010. Le système alimentaire mondial: concepts et méthodes, analyses et dynamiques. Paris : éd. Quae, 565 p. DOI: $10.3917 /$ quae.rasto.2010.01.

Redhead JW, Powney GD, Woodcock BA, Pywell RF. 2020. Effects of future agricultural change scenarios on beneficial insects. Journal of Environmental Management 265: 110550. DOI: 10.1016/j.jenvman.2020.110550.

Rinninella E, Cintoni M, Raoul P, Lopetuso LR, Scaldaferri F, Pulcini $\mathrm{G}$, et al. 2019. Health Gut Microbiota. Nutrients 1-23.

Roth J, Qiang X, Marbán L, Redelt H, Lowell BC. 2004. The obesity pandemic: where have we been and where are we going? Obesity Research 12(S11): 88S-101S. DOI: 10.1038/oby.2004.273.

Runting RK, Bryan BA, Dee LE, Maseyk FJF, Mandle L, Hamel P, et al. 2017. Incorporating climate change into ecosystem service assessments and decisions: a review. Global Change Biology 23(1): 28-41. DOI: $10.1111 / \mathrm{gcb} .13457$.

Schneider A, Huyghe C. 2015. Les légumineuses pour des systèmes agricoles et alimentaires durables. Paris : éd. Quae, 512 p. DOI: 10.35690/978-2-7592-2335-0.

Schmitt E, Galli F, Menozzi D, Maye D, Touzard JM, Marescotti A, et al. 2017. Comparing the sustainability of local and global food products in Europe. Journal of Cleaner Production 165: 346-359. DOI: $10.1016 /$ j.jclepro.2017.07.03:h.

Sirami C, Gross N, Baillod AB, Bertrand C, Carrié R, Hass A, et al. 2019. Increasing crop heterogeneity enhances multitrophic diversity across agricultural regions. Proceedings of the National Academy of Sciences 116(33): 16442-16447. DOI: 10.1073/ pnas. 1906419116.

Solagro. 2014. Afterres 2050. Un scénario soutenable pour l'agriculture et l'utilisation des terres en France à l'horizon 2050. Toulouse : éd. Solagro, 63 p. 
Sommer F, Anderson JM, Bharti R, Raes J, Rosenstiel P. 2017. The resilience of the intestinal microbiota influences health and disease. Nature Reviews Microbiology 15: 630-638. DOI: 10.1038/ nrmicro.2017.58.

Sonnenburg ED, Sonnenburg JL. 2019. The ancestral and industrialized gut microbiota and implications for human health. Nature Reviews Microbiology 17(6): 383-390. DOI: 10.1038/s41579-0190191-8.

Szerencsits M, Weinberger C, Kuderna M, Feichtinger F, Erhart E, Maier S. 2016. Biogas from Cover Crops and Field Residues: Effects on Soil, Water, Climate and Ecological Footprint. International Journal of Environmental and Ecological Engineering 9(4): 413-416.

Therond O, Duru M, Roger-Estrade J, Richard G. 2017. A new analytical framework of farming system and agriculture model diversities: a review. Agronomy for Sustainable Development 37 (21). DOI: 10.1007/s13593-017-0429-7.

Tilman D, Clark M. 2014. Global diets link environmental sustainability and human health. Nature 515(7528): 518-522. DOI: $10.1038 /$ nature13959.

Tirado MC, Clarke R, Jaykus LA, McQuatters-Gollop A, Frank JM. 2010. Climate change and food safety: A review. Food Research International 43(7): 1745-1765. DOI: $10.1016 / \mathrm{j}$.foo dres.2010.07.003.

Toth A, Rendall S, Reitsma F. 2016. Resilient food systems: a qualitative tool for measuring food resilience. Urban Ecosystems 19(1): 19-43. DOI: 10.1007/s11252-015-0489-x.

van Bruggen AHC, He MM, Shin K, Mai V, Jeong C, Finckh MR, et al. 2018. Environmental and health effects of the herbicide glyphosate. Science of The Total Environment 616-617: 255-268. DOI: $10.1016 /$ j.scitotenv.2017.10.309

van de Guchte M, Blottière HM, Doré J. 2018. Humans as holobionts: implications for prevention and therapy. Microbiome 6(1): 81 . DOI: $10.1186 / \mathrm{s} 40168-018-0466-8$.

van den Elsen LWJ, Poyntz HC, Weyrich S, Young W, Forbes-Blom EE. 2017. Embracing the gut microbiota: The new frontier for inflammatory and infectious diseases. Clinical and Translational Immunology 6(1). DOI: 10.1038/cti.2016.91.
Wang L, Li X. 2019. Steering soil microbiome to enhance soil system resilience. Critical Reviews in Microbiology 45(5-6): 743-753. DOI: 10.1080/1040841X.2019.1700906.

Weind I, Ost M, Wiedmer P, Schreiner M, Neugart S, Klopsch R, et al. 2020. Sustainable food protein supply reconciling human and ecosystem health: A Leibniz Consensus. Global Food Security 25: 100367. DOI: $10.1016 /$ j.gfs.2020.100367.

Weishaupt A, Ekardt F, Garske B, Stubenrauch J, Wieding J. 2020. Land use, livestock, quantity governance, and economic instruments-sustainability beyond big livestock herds and fossil fuels. Sustainability (Switzerland) 12(5). DOI: 10.3390/su12052053.

Wezel A, Casagrande M, Celette F, Vian JF, Ferrer A, Peigné J. 2014. Agroecological practices for sustainable agriculture. A review. Agronomy for Sustainable Development 34(1): 1-20. DOI: 10.1007/s13593-013-0180-7.

Yuan X, Pan Z, Jin C, Ni Y, Fu Z, Jin Y. 2019. Gut microbiota: An underestimated and unintended recipient for pesticide-induced toxicity. Chemosphere 227: 425-434. DOI: 10.1016/j.chemos phere.2019.04.088.

Zhang J, Heijden MGA., van der Zhang F, Bender SF. 2020. Soil biodiversity and crop diversification are vital components of healthy soils and agricultural sustainability. Frontiers of Agricultural Science and Engineering 7(3): 236. DOI: 10.15302/j-fase2020336.

Zhou B, Lu Y, Hajifathalian K, Bentham J, Di Cesare M, Danaei G, et al. 2016. Worldwide trends in diabetes since 1980: A pooled analysis of 751 population-based studies with 4.4 million participants. The Lancet 387(10027): 1513-1530. DOI: $10.1016 /$ S0140-6736(16)00618-8.

Zhu G, Zhao Y, Zhu D, Gillings M, Penuelas J, Ok YS, et al. 2019. Soil biota, antimicrobial resistance and planetary health. Environment International 131(July): 105059. DOI: 10.1016/j. envint.2019.105059.

Zimmerer KS, de Haan S, Jones AD, Creed-Kanashiro H, Tello M, Carrasco M, et al. 2019. The biodiversity of food and agriculture (Agrobiodiversity) in the anthropocene: Research advances and conceptual framework. Anthropocene 25: 100192. DOI: 10.1016/j. ancene.2019.100192.

Citation de l'article : Duru M, Le Bras C. 2020. Crises environnementales et sanitaires : des maladies de l'anthropocène qui appellent à refonder notre système alimentaire. Cah. Agric. 29: 34. 